\title{
Transmission properties of van der Waals materials for terahertz time-domain spectroscopy applications
}

Cite as: AIP Conference Proceedings 2300, 020111 (2020); https://doi.org/10.1063/5.0031718

Published Online: 08 December 2020

Polina Shaban, Egor Oparin, Maria Zhukova, Benjamin Hogan, Evgeniya Kovalska, Anna Baldycheva, and Anton Tsypkin

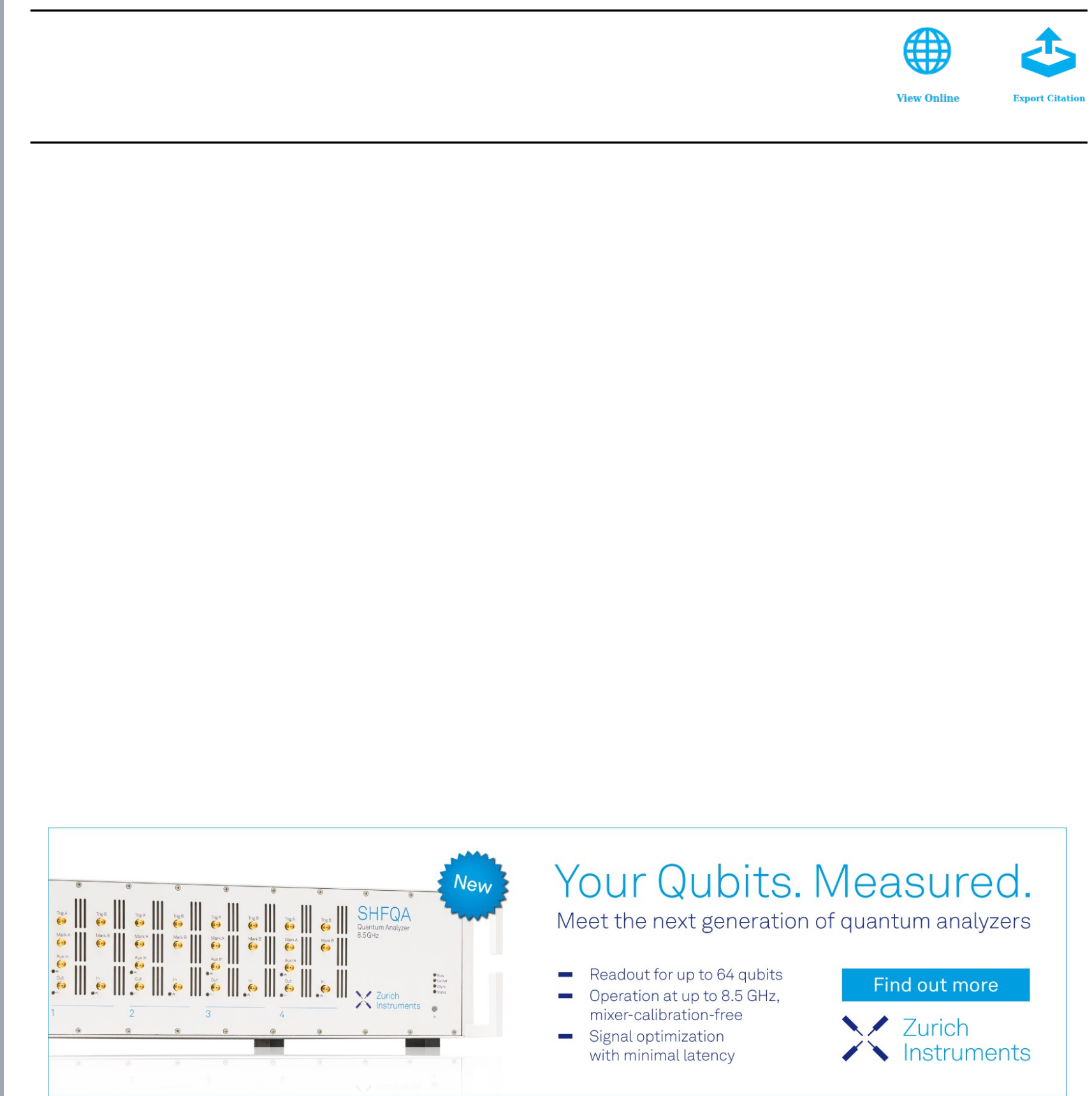




\title{
Transmission Properties Of Van der Waals Materials For Terahertz Time-Domain Spectroscopy Applications
}

\author{
Polina Shaban, ${ }^{1, \text { a) }}$ Egor Oparin, ${ }^{1}$ Maria Zhukova, ${ }^{1}$ Benjamin Hogan,, 3 \\ Evgeniya Kovalska, ${ }^{2,4}$ Anna Baldycheva, ${ }^{2}$ and Anton Tsypkin ${ }^{1}$ \\ 1) ITMO University, Kronverksky Pr., St. Petersburg, 197101, Russia. \\ ${ }^{2)}$ University of Exeter, North Park Road, Exeter, EX4 4QF, UK \\ ${ }^{3)}$ University of Oulu, 90570 Oulu, Finland \\ 4) University of Chemistry and Technology, Praha, 166 28, Czech Republic \\ ${ }^{a)}$ Corresponding author: polinashabanan@gmail.com
}

\begin{abstract}
This work contains results from studying the electro-optical properties of bulk and few-layered Van der Waals materials including intercalated graphene, phosphorene, and tungsten disulfide thin films. Different production methods and substrates are considered. The objective of the research is to assess the relevance of application of these materials in terahertz (THz) time-domain spectroscopy (TDS). Therefore, the study is conducted in the visible, NIR (near-infrared) and THz frequency ranges as the most critically in need of research when searching for effective materials for TDS.
\end{abstract}

\section{INTRODUCTION}

\section{Terahertz time-domain spectroscopy}

Terahertz time-domain spectroscopy has a wide variety of applications including: fundamental physics, imaging, non-destructive testing, bio-medicine, security systems, wireless networks, and others [1]. However there is still a lack of effective components for the generation, detection, and modulation of $\mathrm{THz}$ radiation, encompassing both active and passive elements. Hence, finding applicable materials for such components within THz TDS systems is of great interest. The determination of terahertz time-domain spectroscopy applicability requires studying materials' properties not only in the terahertz frequency range, it is strongly connected with visible and NIR ranges, as the terahertz radiation is typically excited by femtosecond pulses with such wavelengths. Therefore, studying the electrooptical properties of candidate materials in all three ranges is extremely important.

\section{Materials}

Van der Waals layered materials with strong in-plane covalent bonding and weak interlayer (Van der Waals) interactions could be a promising candidate for application in THz TDS. Van der Waals materials can be readily reduced to few layer or monolayer thicknesses through exfoliation, or can be epitaxially grown layer-by-layer. Materials with low layer numbers (below a few layer limit) are considered to be so-called two-dimensional materials. Examples include graphene and its functionalised derivatives, phosphorene, and transition metal dichalcogenides considered in this work. These materials could be effectively implemented into various plasmonic and opto-electronic systems, being compact and often having properties controlled by the number of layers.

Graphene, a zero-bandgap semiconductor, was presented as the first experimentally realised 2D material comprising a single atomic layer in thickness and has already been studied extensively. It has demonstrated high potential for use in terahertz technologies due to its extraordinary carrier transport properties and symmetric conical band structure [2]. Optical excitation transitions in graphene have been studied by various experimental groups, confirming theoretical expectations. However, graphene can also be functionalised. Various chemical groups can either be bonded to the surface or intercalated between layers in order to change the material's properties. Here we investigate the transmission properties of $\mathrm{FeCl}_{3}$ intercalated graphene layers.

Phosphorene, a single layer of black phosphorus (BP), is a more recent addition to the family of layered materials and posseses tremendous electronic properties, such as high carrier mobility, strong light-matter interactions, unique anisotropic physical properties, and a broadband tunable bandgap. In contrast to semi-metallic graphene, BP is a direct bandgap semiconductor. Its layer-number-dependent bandgap is narrower than that of transition metal dichalcogenides

5th International Conference on Metamaterials and Nanophotonics METANANO 2020

AIP Conf. Proc. 2300, 020111-1-020111-4; https://doi.org/10.1063/5.0031718

Published by AIP Publishing. 978-0-7354-4034-0/\$30.00 
(TMDs) [3]. Atomically thin TMDs, such as $\mathrm{WS}_{2}, \mathrm{WSe}_{2}, \mathrm{MoS}_{2}$, and $\mathrm{MoSe}_{2}$ have been common targets of research in the past decade [4]. They have some unique properties, such as tightly bound excitons with binding energies of around several hundred meV [4]. Here the properties of thin tungsten disulfide $\mathrm{WS}_{2}$ films combined with hexagonal boron nitride $(h$-BN) were investigated.

\section{METHODS}

The graphene-based samples (monolayer, few layers - 5-6 atomic layers and multilayers - 50-60 atomic layers) were synthesised on metallic (either copper or nickel) catalysts using a chemical vapour deposition (CVD) system and methane as a carbon source. The few layer and multilayer graphene samples were then intercalated with $\mathrm{FeCl}_{3}$ vapours in a CVD system, using an established process within a three zone furnace $[5,6,7]$. The intercalated samples were transferred to glass, sapphire and flexible polyimide (Kapton) substrates with $1 \mathrm{~mm}, 0.8 \mathrm{~mm}$ and $0.125 \mathrm{~mm}$ thicknesses, respectively [8].

Phosphorene samples were fabricated by a novel controlled low-potential electrochemical exfoliation method in a non-aqueous oxygen-free medium for producing high-quality few-layer micron lateral size phosphorene with 100\% yield and with minimized surface oxidation [9]. Phosphorene layers from the solution were transferred by an isopropyl alcohol (IPA)-assisted direct transfer [10] to substrates such as Kapton, glass and quartz. Thickness of the resulting black phosphorus films was $50 \mathrm{~nm}$ and $150 \mathrm{~nm}$ (termed thin and thick respectively herein).

$\mathrm{WS}_{2}$ films were fabricated from liquid crystalline tungsten disulfide dispersions with a concentration of $10 \mathrm{mg} \cdot \mathrm{mL}^{-1}$ and transferred to polyethylene terephthalate (PET) substrates. The film thicknesses were determined to be around 10 $\mu \mathrm{m}$ [11]. Hexagonal boron nitride films were prepared by the same method as for $\mathrm{WS}_{2}$. Mixed $\mathrm{WS}_{2}$ and $h$-BN films were made by using a 50/50 mixture of the original dispersions and following the same transfer method again.

The transmission properties of the Van der Waals materials samples were studied by using absorption spectroscopy in VIS-NIR ranges and terahertz time-domain spectroscopy [8] in the 0.1-1 THz range.

\section{RESULTS}

\section{Graphene-based samples}
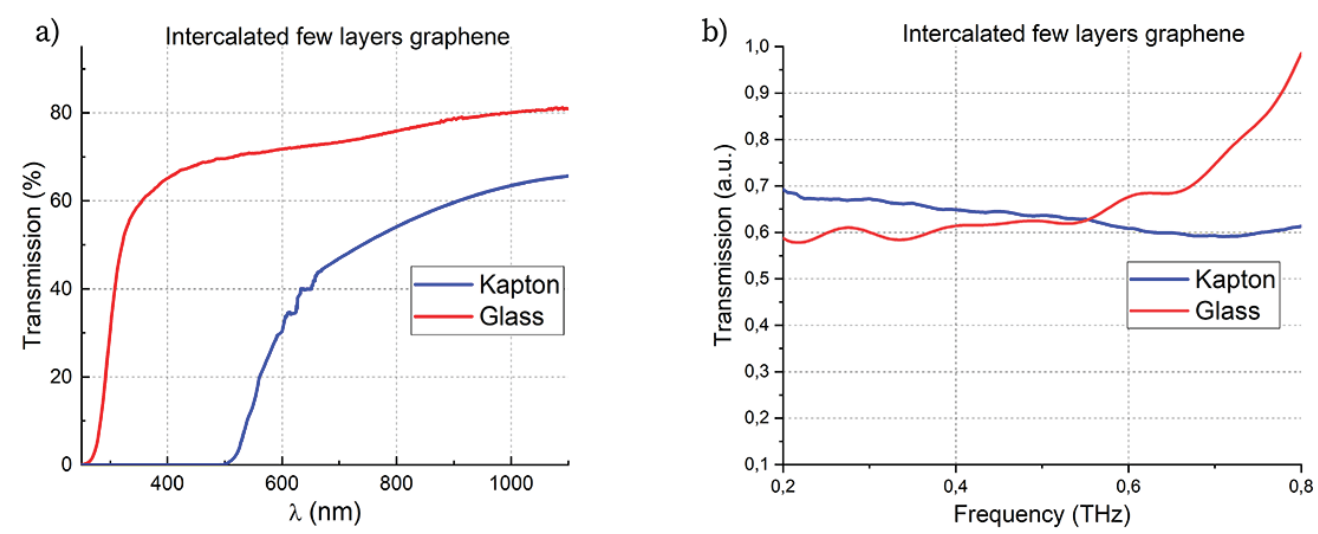

FIGURE 1. Transmission spectra of glass and Kapton substrates with few (5) layer intercalated graphene in visible - NIR (a) and terahertz (b) ranges.

Results of graphene transmission measurements in the VIS-NIR and terahertz ranges are shown in Figure 1. It can be observed, that despite the small number of atomic layers, graphene demonstrates significant absorption of around $20 \%$ (glass) and 10\%(kapton) in the 400-1100 nm range. In the terahertz range thin films are almost transparent, while thick samples absorb about $60 \%$ of incident radiation. 


\section{Phosphorene-based samples}

Results of phosphorene transmission measurements in the VIS-NIR and THz ranges are shown in Figure 2. In the $300-1100$ range absorption of the thin layer is about $10 \%$ and for the thick layer about $50-60 \%$. The results are generally close to those for the graphene samples. In the terahertz range, transmittance is close to $100 \%$ for glass and quartz substrates in the entire range, and for Kapton at frequencies below $\approx 0.6 \mathrm{THz}$. For frequencies greater than 0.6 $\mathrm{THz}$, the transmittance rapidly decreases.

Fabry-Perot oscillations in thin films can be observed in the transmission spectrum of the pure Kapton substrate. The addition of the black phosphorus film causes the disappearance of such oscillations and, for the thin layer sample, the transmission notably increases.

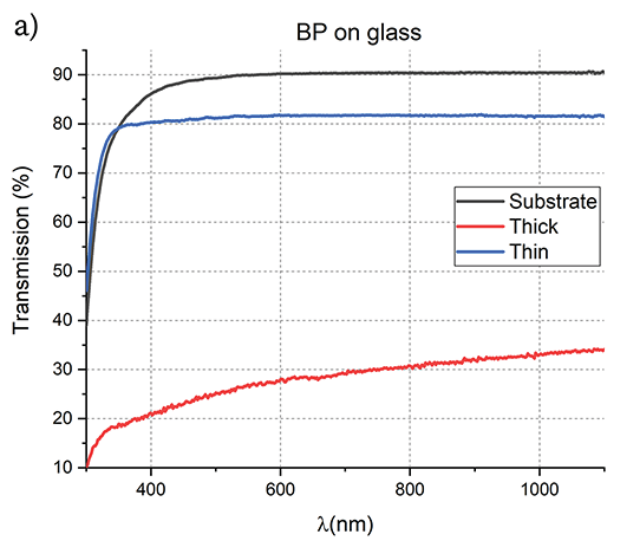

c)

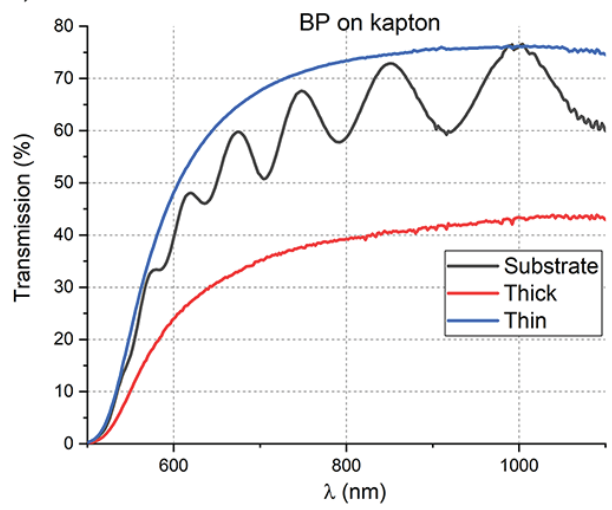

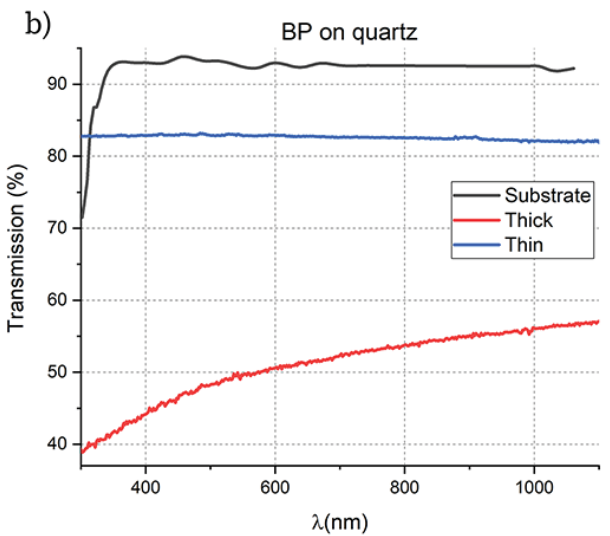

d)

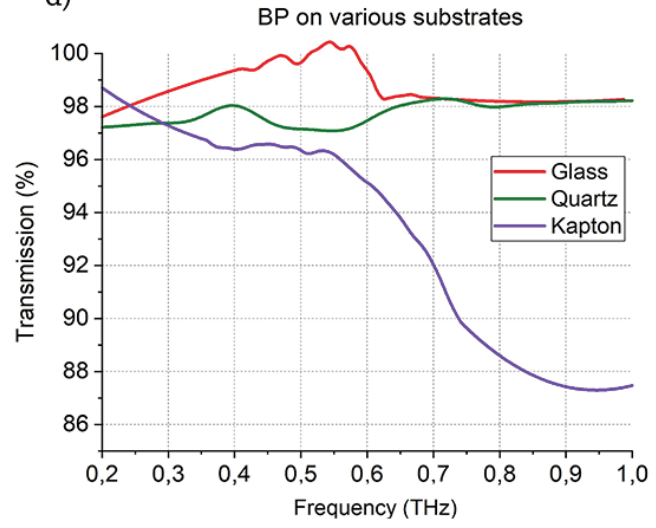

FIGURE 2. Transmission spectra of black phosphorus on glass in the visible-NIR range (a), transmission spectra of black phosphorus on quartz in the visible-NIR range (b), transmission spectra of black phosphorus on Kapton in the visible-NIR range (c) and the transmission spectra of black phosphorus on various substrates in the THz range (d).

\section{Tungsten disulfide-based samples}

Transmission spectra of $\mathrm{WS}_{2}, h$-BN and a $\mathrm{WS}_{2} / h$-BN mixture on PET substrates in the VIS-NIR and terahertz ranges are given in Figure 3. In the 400-1000 nm range absorption of $W S_{2}$ is about $60 \%$. For $h$-BN and $\mathrm{WS}_{2}$ combined with $h$-BN, the transmittance is quite small - about $5-10 \%$. In the terahertz range $\mathrm{WS}_{2}$ on the PET substrate is almost transparent, the transmittance is $\sim 90 \%$. 

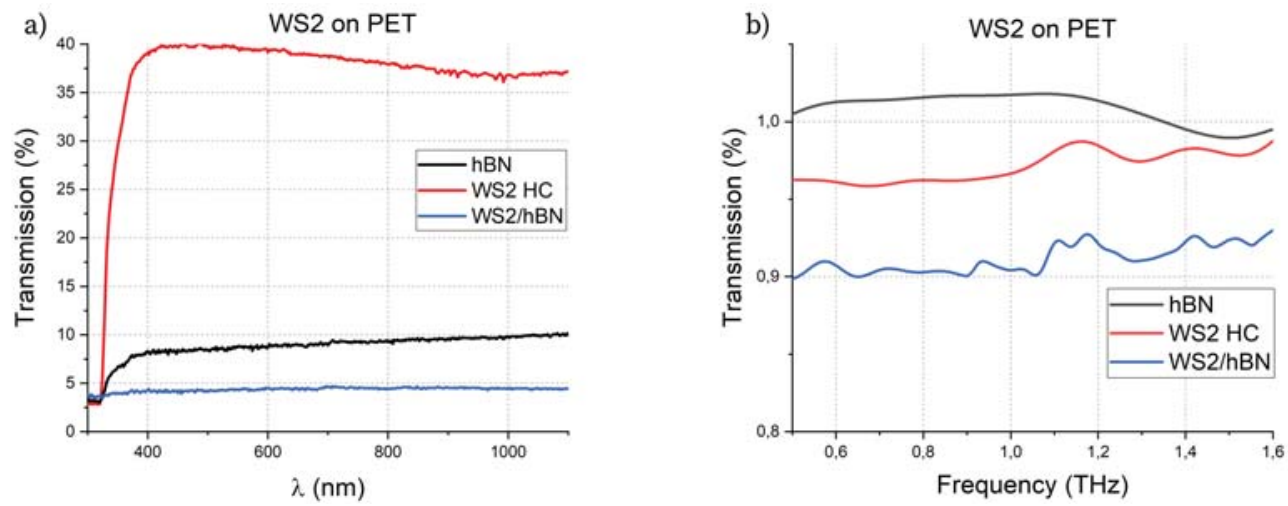

FIGURE 3. Transmission spectra of $\mathrm{WS}_{2}, h$-BN, $\mathrm{WS}_{2} / \mathrm{hBN}$ on PET in the visible-IR range (a), and the THz range (b).

\section{CONCLUSION AND PERSPECTIVES}

Transmission properties of Van der Waals layered materials such as intercalated graphene, layered black phosphorus and tungsten disulfide $\left(\mathrm{WS}_{2}\right)$ thin films were investigated. Explicit transmission properties dependence on thickness (amount of layers), fabrication method, structure modification and substrates was investigated. The important question of finding the dependence of carrier parameters and statistics on these listed factors remains open. However, due to their small thicknesses, high absorption and the possibility to easily tune the materials properties by changing the films thickness, we can conclude that the considered layered materials can be effectively applied in terahertz systems.

An important task is the theoretical assessment and calculation of the electro-optical parameters of the specified materials in the $\mathrm{THz}$ frequency range. As graphene has already been investigated for quite a long period of time, its electro-optical properties and carrier parameters and statistics were studied widely. Phosphorene and transition metal dichalcogenides fabricated in new ways are a relatively new field of study, and the combination of this factor with the strong dependence of the fundamental properties on the number of atomic layers and fabrication method leads to a lack of carrier parameters and statistics knowledge, and hence difficulties in theoretical estimations of the electro-optical properties.

\section{REFERENCES}

1. S. D. et al., "The 2017 terahertz science and technology roadmap," Journal of Physics D: Applied Physics (2017).

2. B. Sensale-Rodriguez, R. Yan, M. Kelly, T. Fang, K. Tahy, W. Hwang, D. Jena, L. Liu, and H. Xing, "Broadband graphene terahertz modulators enabled by intraband transitions," Nature Communications (2012).

3. X. Wang and S. Lan, "Optical properties of black phosphorus," Advances in Optics and photonics (2016).

4. J. Gustafson, P. Cunningham, . McCreary, B. Jonker, and L. Hayden, "Ultrafast carrier dynamics of monolayer ws2 via broad-band timeresolved terahertz spectroscopy," The journal of physics chemistry (2019).

5. D. Zhan, L. Sun, Z. Ni, L. Liu, X. F. Fan, Y. Wang, T. Yu, Y. M. Lam, W. Huang, and Z. X. Shen, "Fecl3-based few-layer graphene intercalation compounds: Single linear dispersion electronic band structure and strong charge transfer doping," Advanced Functional Materials (2010).

6. T. Bointon, G. Jones, A. D. Sanctis, R. Hill-Pearce, M. Craciun, and S. Russo, "Large-area functionalized cvd graphene for work function matched transparent electrodes," Scientific Reports (2015).

7. I. Khrapach, F. Withers, T. Bointon, D. Polyushkin, W. Barnes, S. Russo, and M. Craciun., "Novel highly conductive and transparent graphenebased conductors," Advanced Materials (2012).

8. M. Zhukova, B.Hogan, E. Oparin, P. Shaban, Y. Grachev, E. Kovalska, K. Walsh, M. Craciun, A. Baldycheva, and A. Tcypkin, "Transmission properties of fecl3-intercalated graphene and ws2 thin films for terahertz time-domain spectroscopy applications," Nanoscale research letters (2019).

9. E. Kovalska, J. Luxa, T. Hartman, N. Antonatos, P. Shaban, E. Oparin, M. Zhukova, and Z. Sofer, "Non-aqueous solution-processed phosphorene by controlled low-potential electrochemical exfoliation and thin film preparation," Nanoscale (2020).

10. D. Shin, M. Barnes, K. Walsh, D. Dimov, P. Tian, A. Neves, C. Wright, S. Yu, J. Yoo, S. Russo, and M. Craciun, "A new facile route to flexible and semi-transparent electrodes based on water exfoliated graphene and their single-electrode triboelectric nanogenerator," Advanced Materials (2018).

11. B. Hogan, E. Kovalska, M. Zhukova, M. Yildirim, A. Baranov, M. Craciun, and A. Baldycheva, "2d ws 2 liquid crystals: tunable functionality enabling diverse applications," Nanoscale (2019). 